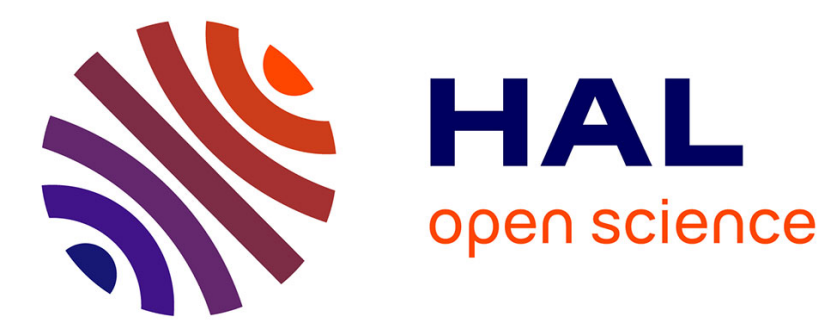

\title{
Building-up virtual optimized mechanism for flame modeling
}

Melody Cailler, Nasser Darabiha, Denis Veynante, Benoit Fiorina

\section{To cite this version:}

Melody Cailler, Nasser Darabiha, Denis Veynante, Benoit Fiorina. Building-up virtual optimized mechanism for flame modeling. Proceedings of the Combustion Institute, 2017, 36 (1), pp.1251 1258. 10.1016/j.proci.2016.05.028 . hal-01541938

\section{HAL Id: hal-01541938 \\ https://hal.science/hal-01541938}

Submitted on 6 Apr 2020

HAL is a multi-disciplinary open access archive for the deposit and dissemination of scientific research documents, whether they are published or not. The documents may come from teaching and research institutions in France or abroad, or from public or private research centers.
L'archive ouverte pluridisciplinaire HAL, est destinée au dépôt et à la diffusion de documents scientifiques de niveau recherche, publiés ou non, émanant des établissements d'enseignement et de recherche français ou étrangers, des laboratoires publics ou privés. 


\title{
Building-up virtual optimized mechanism for flame modeling
}

\author{
Mélody Cailler ${ }^{\mathrm{a}, \mathrm{b}, *}$, Nasser Darabiha ${ }^{\mathrm{a}}$, Denis Veynante ${ }^{\mathrm{a}}$, Benoît Fiorina ${ }^{\mathrm{a}}$ \\ ${ }^{a}$ Laboratoire EM2C, CNRS, CentraleSupélec, Université Paris-Saclay, Grande Voie des Vignes, 92295 Châtenay-Malabry \\ Cedex, France \\ ${ }^{\mathrm{b}}$ SAFRAN Tech, Rue des Jeunes Bois, Châteaufort - CS 80112, 78772 Magny-les-Hameaux, France
}

\begin{abstract}
In most practical applications, a large variety of flame structures varying from premixed, to non-premixed combustion is encountered. To account for such complex combustion regimes, at a reduced computational cost, predictive reduced kinetic schemes are required. Global mechanisms are widely used in the LES context for their low computational cost and easy implementation in CFD codes. However, these mechanisms, built to reproduce global quantities such as laminar flame speed and burnt gas state, are valid on a limited range of flame equivalence ratio, and do not reproduce the local flame structure. A strategy to optimize virtual kinetic schemes based on a genetic algorithm is discussed. The optimization procedure aims at evaluating the set of virtual species properties and reaction rate parameters that best reproduce the temperature profile and laminar flame speed of a reference library of premixed flames. The use of virtual species whose thermodynamic properties are fitted enable the prediction of adiabatic temperature over the whole flammability range. The model also uses a correction for pre-exponential factors to match the laminar flame speed in very lean and rich flames. Furthermore, it is proposed to tabulate the reaction exponent of an intermediate species versus the equivalence ratio in order to adjust the characteristic thickness of the post-flame zone. The resulting kinetic scheme is evaluated on premixed and non-premixed flame configurations. The temperature profiles predicted by the virtual mechanism are in good agreement with these obtained from detailed chemistry calculations for both combustion regimes.
\end{abstract}

\section{Introduction}

Turbulent combustion observed in industrial devices exhibit complex chemical flame structure where both premixed and non-premixed regimes may be observed. To include a description of chemistry in numerical simulations of such turbulent 
flames, methods have been developed to reduce detailed kinetic schemes [1]. Among them chemistry tabulation strategies have shown capabilities to capture the chemical structure of well-identified flames [2-4]. However, they failed when applied to more complex situations, especially where premixed, partially premixed and non-premixed flames structure co-exist [5]. Regarding chemical reduction strategies, skeletal and analytical mechanisms naturally provide an accurate description of the flame structure and intermediate concentrations [6,7]. Nevertheless, their computational costs along with numerical issues due to the reaction rate stiffness remain an important problem for application in practical combustors. An alternative to address fluid/chemistry interactions at a reduced computational cost is the use of global step mechanisms. These ad hoc models composed of one to four steps are built to reproduce global flame properties such as the laminar flame speed, the burnt gas composition or the auto-ignition times [8-11]. However, all these reduced mechanisms are not fitted to describe the structure of the flame.

The objective of the present work is to develop a methodology to address combustion chemistry over both premixed and non-premixed flame regimes. The originality of the method consists in building a chemical mechanism from scratch instead of reducing a detailed scheme. Contrarily to classical reduction methods whose principle is to reduce the number of species and reactions to achieve a certain agreement with the reference mechanism, we suggest to gradually increase the dimensionality of the kinetic scheme so that the quantities of interest are properly described. The novel idea also relies on the use of virtual species with optimized properties instead of the real species (with fixed thermodynamic properties) considered in common optimized pseudo global-schemes $[8,9]$. The reactions between virtual species compose a virtual mechanism that does not represent real chemical reactions but constitute a mathematical architecture designed to retrieve a set of user-defined physical targets. In this article, the virtual scheme is optimized to properly capture the coupling between combustion and the flow field. The following flame properties are to be retrieved:

- flame consumption speed,

- extinction strain rate,

- temperature and heat release profiles.

\section{Methodology for building-up virtual kinetic schemes}

\subsection{Problem formulation}

Combustion is described by a virtual kinetic mechanism composed of $N_{s}$ virtual species interacting through $N_{r}$ virtual reactions represented in the general form:

$\sum_{k=1}^{k=N_{s}} \alpha_{k i}^{\prime} \mathcal{V}_{k} \rightarrow \sum_{k=1}^{k=N_{s}} \alpha_{k i}^{\prime \prime} \mathcal{V}_{k} \quad\left(i=1, \ldots, N_{r}\right)$

where $\alpha_{k i}$ are mass stoichiometric coefficients of the virtual species $\mathcal{V}_{k}$ in the $i$ th reaction.

The proposed strategy aims at optimizing the reaction mechanism to retrieve a set of physical properties. The optimization relies on evaluating:

- the number of reactions and species composing the virtual mechanism,

- thermodynamic and transport properties for virtual species,

- kinetic rate coefficients.

Closures for virtual properties are derived in Section 2.2 whereas kinetics description is discussed in Section 2.3.

\subsection{Virtual species properties}

Thermodynamic properties of virtual species are modeled by standard temperature dependent NASA polynomial:

$$
\begin{aligned}
\frac{c_{p k}^{0}}{R}= & a_{1 k}+a_{2 k} T+a_{3 k} T^{2}+a_{4 k} T^{3}+a_{5 k} T^{4} \\
\frac{h_{k}^{0}}{R T}= & a_{1 k}+\frac{a_{2 k}}{2} T+\frac{a_{3 k}}{3} T^{2}+\frac{a_{4 k}}{4} T^{3} \\
& +\frac{a_{5 k}}{5} T^{4}+\frac{a_{6 k}}{T}
\end{aligned}
$$

where $c_{p k}$ is the heat capacity at constant pressure and $h_{k}$ the enthalpy of the $k$ th species. The superscript 0 refers to the standard-state at one atmosphere. The optimization of coefficients $a_{i k}$ is discussed in Section 3.3. by:

The gas mixture averaged viscosity $\mu$ is modeled

$\mu=\mu_{0}\left(\frac{T}{T_{0}}\right)^{\alpha}$

where $\mu_{0}$ is the reference dynamic viscosity at the reference temperature $T_{0}$. The coefficient $\alpha$ is optimized to fit the dynamic viscosity dependence on temperature.

The mixture thermal conductivity $\lambda$ is evaluated from:

$\lambda=\frac{\mu c_{p}}{P r_{0}}$

where $P r_{0}$ is the Prandtl number and $c_{p}$ is the mixture averaged heat capacity evaluated from the following mixing law: $c_{p}=\sum_{k=1}^{k=N_{s}} c_{p k} Y_{k} . P r_{0}$ is assumed equal to the burnt gases Prandtl number of a stoichiometric mixture at initial temperature $T_{0}$.

Finally, the molecular diffusion term is expressed using a unity Lewis number formalism:

$\frac{\partial}{\partial x_{i}}\left(\rho Y_{k} V_{k}\right)=-\frac{\partial}{\partial x_{i}}\left(\frac{\lambda}{c_{p}} \frac{\partial Y_{k}}{\partial x_{i}}\right)$. 


\subsection{Virtual reactions mechanisms}

We assume that the reactive mixture, composed of fuel $(F)$, oxidizer $(O x)$ and an inert dilutant $(D)$, is transformed into products through a virtual mechanism. Products are modeled by a combination of $N_{p}$ virtual species $P_{k}$. Two virtual mechanism structures are investigated here:

- A one step irreversible reaction named M1:

$$
\alpha_{F} F+\alpha_{O x} O x \rightarrow \sum_{k=1}^{k=N_{P}} \alpha_{P_{k}} P_{k}
$$

- A two-step mechanism called M2 composed of two consecutive reactions. It converts first the reactants into an intermediate species $I$, which is then transformed into products $P_{k}$ :

$$
\begin{aligned}
& \alpha_{F} F+\alpha_{O x} O x \rightarrow \alpha_{I} I \\
& \alpha_{I} I \rightarrow \sum_{k=1}^{k=N_{P}} \alpha_{P_{k}} P_{k}
\end{aligned}
$$

Chemical reaction rates are expressed with Arrhenius type laws. The rate of progress variable $q_{1}$ of the reactions M1-1 and M2-1 is given by:

$q_{1}=A_{1} \exp \left(\frac{-E_{a, 1}}{R T}\right)[F]^{n_{F, 1}}[O x]^{n} O x, 1$

while the rate of progress variable $q_{2}$ of the reaction M2-2 reads:

$q_{2}=A_{2} \exp \left(\frac{-E_{a, 2}}{R T}\right)[I]^{n_{I, 2}}$,

where $A_{i}$ is the pre-exponential factor, $E_{a, i}$ the activation energy of the $i$ th reaction, and $n_{k, i}$ the reaction exponent of the $k$ th species in reaction $i$.

\section{Optimization strategy}

\subsection{Targeted flame properties}

The objectives are to determine the parameters involved in the thermo-chemical and kinetics closures of the virtual mechanism. It should be noted that the current work does not include optimization of species transport properties, assumption of unity Lewis numbers being retained. Thermochemical parameters presented in Section 2.2 are identified to reproduce temperature dependent properties of a reactive fuel/oxidizer/dilutant mixture (from fresh gases to equilibrium conditions). Kinetics parameters introduced in Section 2.3 are selected so that the virtual mechanism reproduces as best as possible a set of flame properties given by a reference database. The reference database is a collection of flame solutions obtained with a detailed chemical scheme. Here premixed flamelets

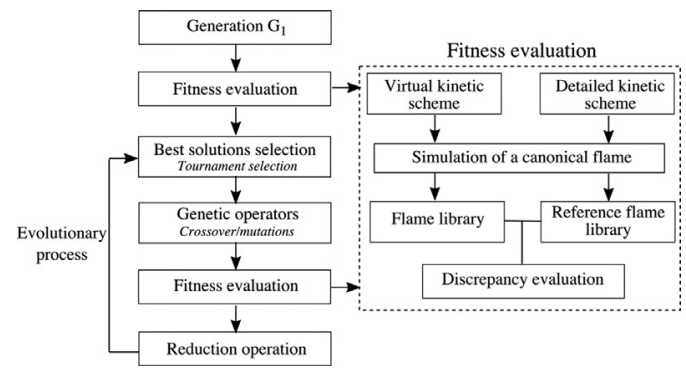

Fig. 1. Schematic procedure of optimization.

are considered but other flame archetypes could be included in this database. The target flame properties selected to capture the flame and its interaction with the flow, are temperature, density and flame consumption speed. The next section describes the optimization algorithm retained to identify these parameters.

\subsection{Optimization algorithm}

Among the various type of optimization techniques available in the literature, genetic algorithms are adapted to combustion kinetic mechanisms [12-14]. Genetic algorithms belong to a family of stochastic methods which explores the domain by modeling biological evolution principles. $\mathrm{N}$ set of parameters (for thermo-chemical or kinetics properties) are randomly initialized to constitute the first generation $G_{1}$, which then undergoes the principle of natural selection through the use of a fitness function. The fitness function compares solutions given by the virtual mechanism against the reference database made of detailed chemical scheme solutions. Heredity laws (crossover and mutation) are then modeled to enable the mixing of qualities of the best individuals and finally improve the prediction capabilities of the kinetic models. Figure 1 illustrates this optimization procedure.

Unlike classical gradient based methods, genetic algorithms are able to handle complex fitness functions featuring highly structured landscapes, noise and insufficient information [15]. Moreover, the balance between space exploration and space exploitation enables genetic algorithms to converge to local extrema contrarily to classical methods which generally converge to global extremum [16].

\subsection{Optimization of virtual species properties}

A first optimization loop is performed to identify the virtual species properties that best reproduce the real mixture thermodynamic properties. The mean properties of the virtual mixture reads: 


$$
\begin{aligned}
\Psi^{v}= & Y_{F}^{v} \Psi_{F}^{v}+Y_{O x}^{v} \Psi_{O x}^{v}+Y_{D}^{v} \Psi_{D}^{v}+Y_{I}^{v} \Psi_{I}^{v} \\
& +\sum_{k=1}^{k=N_{P}} Y_{P_{k}}^{v} \Psi_{P_{k}}^{v}
\end{aligned}
$$

where $\Psi_{k}$ is the thermodynamic properties (here $h_{k}$ and $c_{p k}$ ) of species $k$ and the superscript $v$ denotes virtual species properties or mass fraction.

To retrieve the mixture properties in fresh gases, real thermodynamic properties are attributed to reactant species:

$\Psi_{A}^{v}=\Psi_{A}^{*}, \quad$ for $A=F, D$ and $O x$.

The * superscript denotes reference species properties.

The modeling of burnt gases properties is more complex as hundred of real species are involved in hydrocarbon/air equilibrium composition. An optimization procedure is then required to describe burnt gases properties with a minimal number $N_{P}$ of virtual products $P_{k}$ along with the reactant species present in the burnt gases. Under equilibrium conditions, species mass fractions of virtual products are related to mass stoichiometric coefficients $\alpha_{P_{k}}$ given in Eqs. (M1-1) and (M2-2):

$\left.Y_{P_{k}}^{v}\right|^{e q}=\left.\alpha_{P_{k}} Y_{P}^{v}\right|^{e q}$

where $\left.Y_{P}^{v}\right|^{e q}$ is the total mass fractions of virtual products in fully burnt gases. Both stoichiometric coefficients $\alpha_{P_{k}}$ and thermodynamic properties $\Psi_{P_{k}}^{v}$ (in practice the coefficients $a_{i k}$ defined in Eqs. (2) and (3)) of virtual products are optimized so that the equality $\left.\phi^{v}\right|^{e q}=\left.\phi^{*}\right|^{e q}$ is satisfied for equivalence ratios varying between lean $\left(\phi_{L}\right)$ and rich flammability limits $\left(\phi_{R}\right)$. Note that the following physical constraints are imposed to the optimization algorithm:

$$
\begin{aligned}
& \sum_{k=1}^{k=N_{P}} \alpha_{P_{k}}=1 \\
& 0<\alpha_{P_{k}}<1 \quad \text { for } k \in\left[1, N_{p}\right]
\end{aligned}
$$

Figure 2 compares the adiabatic flame temperature predicted with an optimized product mixture composed of two, three and four optimized virtual species. As a comparison, the equilibrium temperature obtained from real sets of product species: $\left(\mathrm{H}_{2} \mathrm{O} ; \mathrm{CO}_{2}\right),\left(\mathrm{H}_{2} \mathrm{O} ; \mathrm{CO}_{2} ; \mathrm{CO}\right)$ and $\left(\mathrm{H}_{2} \mathrm{O} ; \mathrm{CO}_{2} ; \mathrm{CO}\right.$; $\mathrm{H}_{2}$ ) is shown in Fig. 2, right.

For low numbers of products, the use of virtual species with optimized properties and stoichiometric coefficients is more efficient to recover the burnt gas temperature than using actual species. Indeed, virtual mixtures composed of only two and three products depart from detailed chemistry only near the stoichiometry, while calculations performed with real mixtures greatly overestimate the adiabatic temperature for rich mixtures. When four products are included the proposed strategy reproduces the burnt gas temperature over the whole

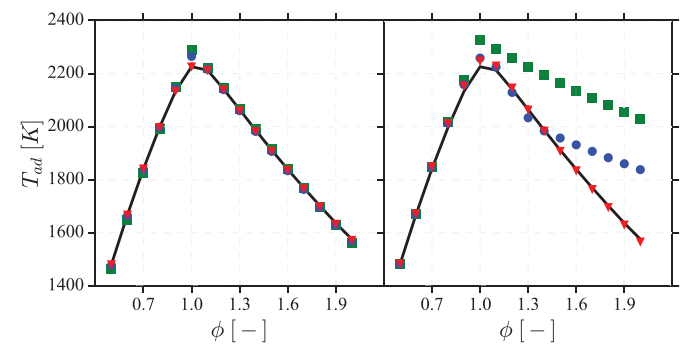

Fig. 2. Adiabatic flame temperature versus equivalence ratio for a premixed methane/air flame at fresh gas temperature $T_{f}=300 \mathrm{~K}$ and pressure $P=1 \mathrm{~atm}$. Left: virtual product mixture, right: real product mixture. Reference equilibrium computation including 53 species [19] (-) and equilibrium calculations including two ( $\square$ ), three $(\bullet)$ and four products $(\nabla)$ are compared.

range of equivalence ratios, while the mixture described by a limited selection of real species slightly overpredicts the temperature near the stoichiometry. The four products mixture is considered for the next calculations.

In the two-step mechanism M2 the intermediate species are completely consumed by the reaction (M2-2). Thermochemical properties of the intermediate species $I$ do not affect the equilibrium condition. However, the standard enthalpy of formation of $I$ species has been chosen to enable an endothermic behavior of reaction M2-2 for rich mixtures by imposing the following constraint:

$\Delta H_{f, I}^{0}<\sum_{k=1}^{N_{p}} \alpha_{P_{k}} \Delta H_{f, k}^{0}$ for $\phi>1.0$

where $\Delta H_{f, I}^{0}=-9.77 M J . \mathrm{kg}^{-1}$ while the products enthalpy evolves between $-10.1 \mathrm{MJ} \mathrm{kg}^{-1}$ and $-8.12 \mathrm{MJ} \mathrm{kg}^{-1}$ from $\phi=0.5$ to $\phi=2.0$. Note that the classical reaction $\mathrm{CO}+\frac{1}{2} \mathrm{O}_{2} \rightarrow \mathrm{CO}_{2}$, considered in many global mechanisms $[8,11]$, cannot be endothermic as $\Delta H_{f, C O}^{0}>\Delta H_{f, \mathrm{CO}_{2}}^{0}$.

\subsection{Optimization of virtual reactions rate parameters}

The second step of the optimization procedure evaluates reaction rate parameters providing the best description of the temperature profiles and laminar flame speed. Reduced low order kinetic schemes (1-4 reactions) cannot describe the laminar flame speed over the all range of equivalence ratios [17]. To overcome this problem a method based on the adjustment of rate coefficients has been proposed $[10,11,18]$. In the present work, the pre-exponential constant of the first reaction is corrected by a function $f_{1}$ depending on the dilutant mass fraction $Y_{D}^{v}$. The correction function $f_{1}$ is optimized to match the laminar flame speed within the flammability limits. For the M2 mechanism, the 
reaction exponent of the intermediate $n_{I, 2}$ is also tabulated as a function of $Y_{D}^{v}$ to control the thickness of the post flame zone and improve the flame structure prediction. Rate of progress variable $q_{1}$ and $q_{2}$ therefore reads:

$q_{1}=A_{1} f_{1}\left(Y_{D}^{v}\right) \exp \left(\frac{-E_{a, 1}}{R T}\right)[F]^{n_{F, 1}}[O x]^{n_{O x, 1}}$,

$q_{2}=A_{2} \exp \left(\frac{-E_{a, 2}}{R T}\right)[I]^{n_{I, 2} f_{2}\left(Y_{D}^{v}\right)}$.

The genetic algorithm presented in Section 3.2 determines the set of reaction rate parameters $\left(A_{i}\right.$, $\left.E_{a, i}, n_{k, i}\right)$ and correction functions $f_{1}$ and $f_{2}$ that best reproduce the temperature profiles and laminar flame speed. This is achieved through the minimization of the fitness function $f$ defined as:

$f=\sum_{i=1}^{N_{c}} w_{1} \frac{\left|S_{L_{i}}^{v}-S_{L_{i}}^{*}\right|}{S_{L_{i}}^{*}}+w_{2} \frac{\left\|T_{i}^{v}-T_{i}^{*}\right\|_{L_{2}}}{\left\|T_{i}^{*}\right\|_{L_{2}}}$,

where $N_{c}$ denotes the number of flamelets in the reference database, $S_{L_{i}}$ and $T_{i}$ respectively represent the laminar flame speed and the temperature profile of the $i$ th flamelet. The factors $w_{1}$ and $w_{2}$ are weights attributed to each objective. Various tests show that $w_{1}=0.1$ and $w_{2}=0.9$ is well adapted to our case.

\section{Results}

The reference data used to calibrate species properties and reaction rate parameters are obtained from equilibrium and 1D-premixed simulations with the GRI3.0 reaction mechanism [19] involving 53 species and 325 reactions. The target premixed flames retained for the kinetic rate optimization step being obtained using detailed transport coefficients include differential diffusion effects. The reference flame library has been generated for a fresh gas temperature $T_{f}=300 \mathrm{~K}$, initial pressure $P=1 \mathrm{~atm}$, and equivalence ratio evolving from lean to rich flammability limit $(\phi \in[0.5,2.0])$. This leads to a learning database composed of $N_{c}=31$ flamelets. To ensure physical values for the optimized coefficients, thermodynamic and kinetic rate coefficients are bounded between user-defined values. The definition intervals have been chosen to be large enough to enable diversity of the solutions and sufficiently restrictive to limit the convergence time of the algorithm. Tests have been performed to check the optimized coefficients sensitivity to the definition intervals.

The optimized kinetic rate parameters are given in Table 1, while the evolution of tabulated functions $f_{1}$ (for reaction M1-1 and M2-1) and $f_{2}$ (for reaction M2-2) is displayed in Fig. 3. Contrarily to previous works $[10,11]$ the correction function $f_{1}$ has also been applied to lean mixtures to perfectly
Table 1

Kinetic rate constants (units: $\mathrm{cm}$, s, cal and mol).

\begin{tabular}{lllll}
\hline Reaction & $A_{i}$ & $E_{a, i}$ & $n_{k, i}$ & \\
\hline (M1-1) & $6.3 \times 10^{17}$ & $35.6 \times 10^{3}$ & $n_{F, 1}$ & 1.64 \\
& & & $n_{O x, 1}$ & 0.92 \\
$(\mathrm{M} 2-1)$ & $1.5 \times 10^{18}$ & $35.6 \times 10^{3}$ & $n_{F, 1}$ & 1.70 \\
& & & $n_{O x, 1}$ & 0.86 \\
$(\mathrm{M} 2-2)$ & $3.9 \times 10^{18}$ & $85.7 \times 10^{3}$ & $n_{I, 2}$ & 2.34 \\
\hline
\end{tabular}

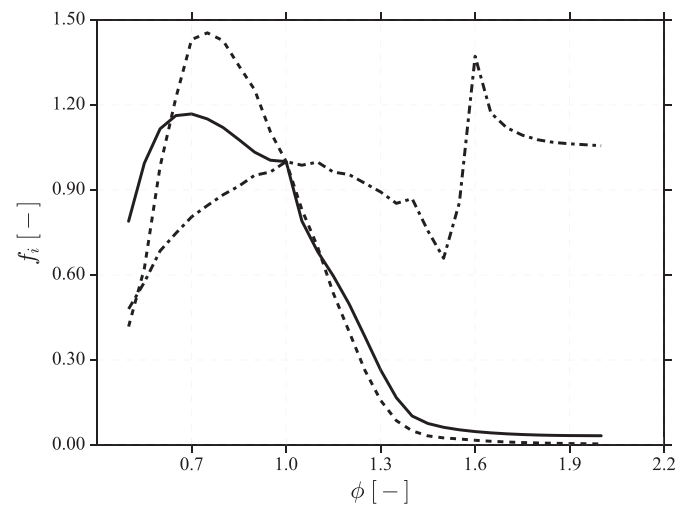

Fig. 3. Evolution of the correction function $f_{i}$ with the equivalence ratio. Function $f_{1}$ for reaction (M1-1) (-), $f_{1}$ for reaction (M2-1) (_- ) and $f_{2}$ for reaction (M2-2) (-.-).

match the laminar flame speed for very lean mixtures $(\phi \in[0.5,0.6])$.

The virtual mechanisms are tested on both freely propagating premixed and counterflow nonpremixed flame configurations. The simulations have been performed with the laminar flame solver REGATH [20].

\subsection{Premixed flames}

To evaluate the capabilities of the optimized virtual mechanisms a set of laminar premixed flames is simulated. Figure 4 shows the laminar flame speed predicted by the detailed kinetic mechanism GRI3.0 [19], the semi-global scheme 2S_CH4_BFER [21], the virtual one- and two-step mechanisms.

For the whole range of equivalence ratios, the optimized virtual schemes M1 and M2 predict accurately the laminar flame speed. The optimization of the correction function $f_{1}$ enables a perfect agreement between the flame speed evaluated from the reduced optimized mechanism and the reference detailed chemistry solutions. Despite the unity Lewis number assumption of the virtual mechanism, the impact of differential diffusion on the laminar flame speed is well described.

A comparison of the spatial evolution of the dimensionless temperature $T^{+}$predicted by the four 


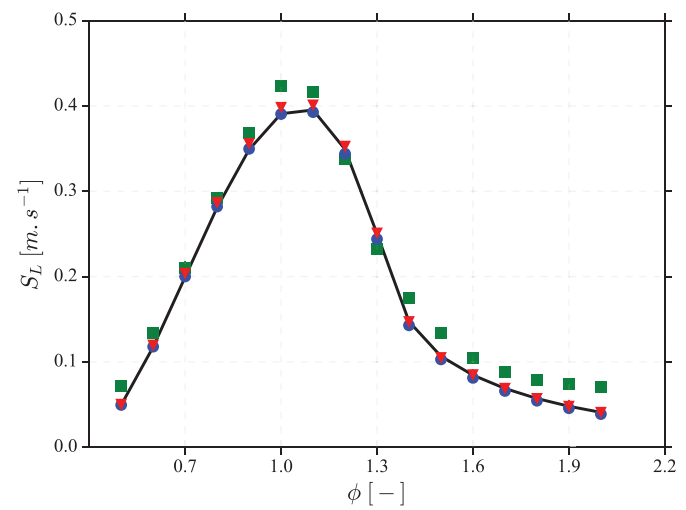

Fig. 4. Laminar flame speed versus equivalence ratio for a premixed methane/air flame at fresh gas temperature $T_{f}=300 \mathrm{~K}$ and pressure $P=1 \mathrm{~atm}$. Detailed chemical mechanism (-), 2S_CH4_BFER scheme (ロ), M1 (•) and M2 $(\nabla)$ mechanisms are compared.

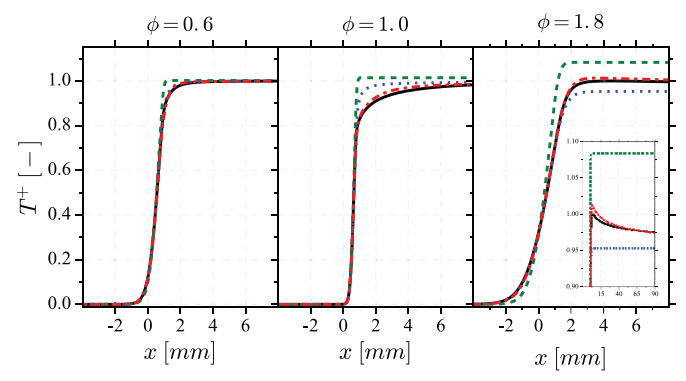

Fig. 5. Spatial evolution of the dimensionless temperature $T^{+}=\frac{T-T_{\min }^{*}}{T_{\max }^{*}-T_{\min }^{*}}$ for a $1 \mathrm{D}$ premixed methane/air flame at fresh gas temperature $T_{f}=300 \mathrm{~K}$ and pressure $P=1 \mathrm{~atm}$. Detailed chemical mechanism ( $)$, 2S_CH4_BFER scheme (_- ), M1 (...) and M2 (_. . ) virtual mechanisms are compared.

mechanisms for different equivalence ratios is presented in Fig. 5.

For lean condition at $\phi=0.6$ (Fig. 5 left), the two virtual mechanisms provide a correct description of the temperature evolution. However, at $\phi=1$, discrepancies arise between the two models. Contrarily to the M2 scheme the optimized onestep mechanism M1 fails to predict the two layers flame structure composed of a thin fuel oxidation layer followed by a thicker $\mathrm{CO}$ oxidation layer [22]. The one step mechanism featuring a unique characteristic time is indeed not able to predict both the two layers flame structure observed for $\phi \in[0.7$, 1.3], and the single-layer structure taking place for very lean $(\phi \in[0.5,0.7])$ and rich $(\phi \in[1.3,2.0])$ mixtures. Regarding the M2 scheme, the use of a tabulated intermediate reaction order versus the dilutant $\left(N_{2}\right)$ mass fraction enables the adjustment of the characteristic thickness of the second layer and a proper description of both flame structure types.

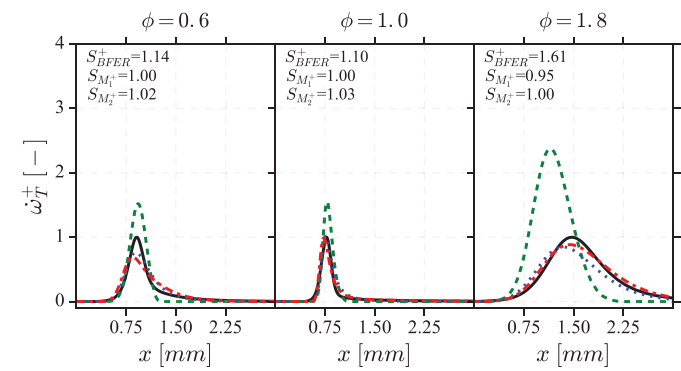

Fig. 6. Spatial evolution of the dimensionless heat release $\dot{\omega}_{T}^{+}=\frac{\dot{\omega}_{T}}{\dot{\omega}_{T_{\max }^{*}}^{*}}$ for a 1D premixed methane/air flame at fresh gas temperature $T_{f}=300 \mathrm{~K}$ and pressure $P=1 \mathrm{~atm}$. Dimensionless integrals of the heat release over the flame front $S^{+}=\frac{\int_{x} H R d x}{\int_{x} H R^{*} d x}$ predicted by the three reduced models are also given. Detailed chemical mechanism ( $)$, 2S_CH4_BFER scheme (_- ), M1 (...) and M2 (_. . ) virtual mechanisms are compared.

The 2S_CH4_BFER scheme predicts a very thin post flame region where the temperature is overestimated. Detailed chemistry simulations show that for rich injection conditions (for $\phi>1.4$ ), the maximum flame temperature reached in the flame front is significantly higher (see inset of Fig. 5 right) than the equilibrium temperature $T_{a d}$ obtained in fully burnt gases $(x \rightarrow+\infty)$. This phenomenon is well reproduced by the two-step mechanism, where the second reaction acts as an endothermic reaction, hence predicting a decrease of temperature in the premixed flame front. The 2S_CH4_BFER scheme is not optimized to capture this effect and predicts a rapid reach of the equilibrium. The M1 mechanism, being in essence not able to reproduce such temperature decrease, also reaches directly the equilibrium temperature.

To evaluate the capabilities of the virtual mechanism to describe the local heat release is mandatory, even though this quantity is not an explicit target of the optimization process. Figure 6 illustrates the dimensionless heat release evolution along the flame for the four reduced mechanisms. The dimensionless integral of the heat release over the flame, $S^{+}$, is also displayed in Fig. 6. For stoichiometric and rich conditions, the optimized virtual mechanisms correctly predict the heat release profiles in terms of both amplitude and thickness. For lean injection conditions, the amplitude of the maximum heat release is slightly under-estimated. According to the 1-D premixed flame theory [23], the heat release integral across the flame front is related to the consumption speed and mixture thermodynamic properties $\left(c_{p}\right.$ and $\left.h\right)$ in fresh and hot gases. As these last quantities are successfully described by the virtual optimized mechanisms, the heat release integral is well recovered. It should be noted that the 2S_CH4_BFER scheme based on a limited number of real species does not predict correctly the 


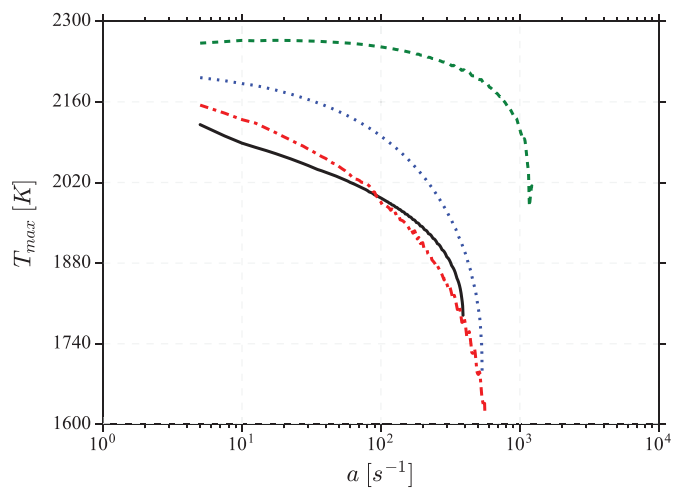

Fig. 7. Evolution with the strain a of the peak temperature obtained in a 1D counterflow methane/air flame at fresh gas temperature $T_{f}=300 \mathrm{~K}$ and pressure $P=1 \mathrm{~atm}$. Detailed chemical mechanism ( $)$, 2S_CH4_BFER scheme (_-), M1 (...) and M2 (_- _) virtual mechanisms are compared.

thermodynamic state of burnt gases and is not able to capture this key quantity. Moreover the globalstep mechanism fails to reproduce the local heat release for the whole range of equivalence ratios.

\subsection{Diffusion flames}

The virtual optimized schemes are now tested for diffusion flames. A set of counterflow flames have been simulated for strain rates varying from $a=15 \mathrm{~s}^{-1}$ to $a_{c}$ the strain rate at which the diffusion flame is close to quenching. The variation with the strain rate of the peak temperature obtained in the flame front is presented in Fig. 7. The optimized one-step mechanism M1 and the 2S_CH4_BFER scheme both overpredict the maximum temperature. On the contrary, the two-step virtual mechanism is able to predict the level of maximum temperature obtained in the diffusion flame front. The two virtual optimized mechanisms slightly overpredict the extinction strain rate, while the 2S_CH4_BFER mechanism is almost not affected by the strain and overpredicts from an order of magnitude the quenching strain limit.

The temperature profiles predicted by the detailed kinetic mechanism and the reduced schemes are displayed in Fig. 8. The M1 mechanism and the 2S_CH4_BFER scheme overpredict the temperature for the whole range of strain rates. The results provided by the two-step virtual mechanism are close to the reference solution, except at low strain rates where a slight departure is observed. For all reduced models and level of strain rate the position of the maximum temperature is shifted towards the fuel side. The discrepancies observed between reference and virtual schemes solutions may be related to the unity Lewis number assumption. As the diffusion flame archetype is not included in

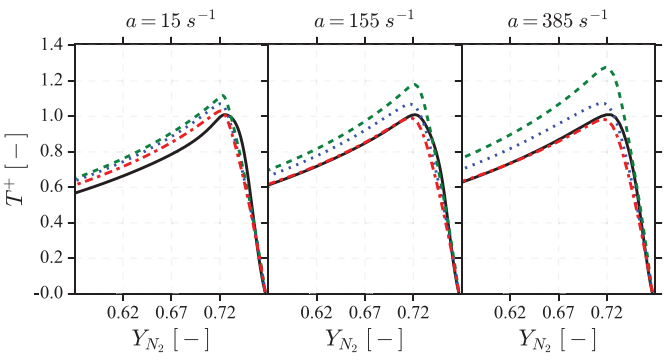

Fig. 8. Dimensionless temperature $T^{+}$evolution with the dilutant mass fraction $Y_{N_{2}}$ for a 1D counterflow methane/air flame at fresh gas temperature $T_{f}=300 \mathrm{~K}$ and pressure $P=1 \mathrm{~atm}$. Detailed chemical mechanism (-), 2S_CH4_BFER scheme (--), M1 (...) and M2 (- - - ) virtual mechanisms are compared.

the targeted flames library, the effects of differential diffusion under strain rate are not fully captured with the transport model retained. An improvement would be to add the counterflow flames in the reference database, and identify the virtual species transport properties $\left(L e_{k}\right)$ that best capture differential diffusion effects under both strained and unstrained configurations.

\section{Conclusions}

A strategy to build virtual kinetic mechanisms has been presented. This method relies on both the optimization of species properties and kinetic rate parameters to retrieve temperature profiles and laminar flame speeds of a collection of premixed flames generated with a detailed kinetic mechanism. Two virtual kinetic schemes have been developed and evaluated. Comparisons of the reduced schemes with the detailed kinetic model GRI3.0 show that both the one- and two-step mechanisms reproduce global flame properties $\left(S_{L}\right.$ and $\left.T_{a d}\right)$ over the whole flammability range. In terms of flame structure, the two-step scheme provides a good description of the premixed flame temperature profiles. Though not optimized for the description of non-premixed combustion, the virtual two-step mechanism turns out to be efficient for the prediction of the temperature profile for strain rates varying from almost zero to the quenching limit. These results highlight the capabilities of the proposed strategy to describe the flame/flow field interaction for both premixed and non-premixed combustion. Furthermore, it should be noted that compared with the detailed scheme a reduction of computational time by a factor of 200 is observed with the optimized virtual schemes.

Next objectives include the investigation of the virtual kinetic scheme suitability in a wider range of operating conditions (pressure, fresh gas temperature, heat losses, etc.). If required, the number of 
species and reactions will be increased to enlarge the validity domain. Also, the methodology will be applied to more complex fuel such as kerosene for which the computational gain will be further increased. Finally, this development will be extended to the description of pollutant formation.

\section{Acknowledgments}

Safran Tech is gratefully acknowledged for supporting M. Cailler's Ph.D. work. The author also would like to acknowledge Benedetta Franzelli for discussions about the models.

\section{References}

[1] B. Fiorina, D. Veynante, S. Candel, Flow Turbul. Combust. 94 (2014) 3-42.

[2] O. Gicquel, N. Darabiha, D. Thévenin, Proc. Combust. Ins 28 (2000) 1901-1908.

[3] J.A. van Oijen, F.A. Lammers, L.P.H. de Goey, Combust. Flame 127 (2001) 2124-2134.

[4] N. Peters, Prog. Energy Combust. Sci. 10 (1984) 319-339.

[5] B. Fiorina, O. Gicquel, L. Vervisch, S. Carpentier, N. Darabiha, Combust. Flame 140 (2005) 147-160.

[6] J. Luche, Elaboration of Reduced Kinetic Models of Combustion. Application to a Kerosene Mechanism, LCSR Orléans, 2003 (Ph.D. thesis).

[7] T. Lu, C. Law, 5th US Combustion Meeting (2007).

[8] C. Westbrook, F. Dryer, Combust. Sci. Technol. 27 (1981) 31-43.

[9] W.P. Jones, R.P. Lindstedt, Combust. Flame 73 (1988) 222-233.
[10] E. Fernandez-Tarrazo, A. Sanchez, A. Linan, F. Williams, Combust. Flame 147 (2006) 32-38.

[11] B. Franzelli, E. Riber, M. Sanjosé, T. Poinsot, Combust. Flame 157 (2010) 1364-1373.

[12] W. Polifke, W. Geng, K. Dobbeling, Combust. Flame 135 (1998) 119-135.

[13] S.D. Harris, L. Elliott, D.B. Ingham, M. Pourkashanian, C.W. Wilson, Comput. Methods Appl. Mech. Eng. 190 (2000) 1065-1090.

[14] B. Farcy, A. Abou-Taouk, L. Vervisch, P. Domingo, N. Perret, Fuel 118 (2014) 291-299.

[15] Z. Michalewicz, Genetic Algorithms+Data Structures=Evolution Programs, third ed., Springer Berlin Heidelberg, 1996.

[16] L. Elliott, D.B. Ingham, A.G. Kyne, N.S. Mera, M. Pourkashanian, C.W. Wilson, Prog. Energy Combust. Sci. 30 (2004) 297-328.

[17] J.P. Légier, Simulation Numérique des Instabilités de Combustion Dans les Foyers Aéronautiques, INP Toulouse, 2001 (Ph.D. thesis).

[18] A. Roux, L.Y.M. Gicquel, S. Reichstadt, et al., Combust. Flame 157 (2010) 176-191.

[19] G.P. Smith, D.M. Golden, M. Frenklach, N.W. Moriarty, B. Eiteneer, M. Goldenberg, C.T. Bowman, R.K. Hanson, S. Song, W.C. Gardiner, V.V. Lissianski, Z. Qin, GRI 3.0, Gas Research Institute, Chicago, IL, 2011. http://www.me.berkeley.edu/gri_ mech.

[20] B. Franzelli, B. Fiorina, N. Darabiha, Proc. Combust. Inst. 34 (2013) 1659-1666.

[21] B. Franzelli, E. Riber, L.Y.M. Gicquel, T. Poinsot, Combust. Flame 159 (2012) 621-637.

[22] M. Bui-Pham, K. Seshadri, F.A. Williams, Combust. Flame 89 (1992) 343-362.

[23] T. Poinsot, D. Veynante, Theoretical and Numerical Combsution, third ed., 2012. 